\title{
What are the impacts of manipulating grazing and browsing by ungulates on plants and invertebrates in temperate and boreal forests? A systematic review protocol
}

Claes Bernes $^{1^{*}}$, Bengt Gunnar Jonsson ${ }^{2}$, Kaisa Junninen ${ }^{3,4}$, Asko Lõhmus $^{5}$, Ellen Macdonald ${ }^{6}$, Jörg Müller ${ }^{7}$ and Jennie Sandström²

\begin{abstract}
Background: Livestock grazing and 'overabundance' of large wild herbivores in forested areas have long been perceived as conflicting with the aims of both silviculture and forest conservation; however, certain kinds of herbivory can help to maintain habitat values in forest ecosystems. Management of grazing/browsing in protected forests can, therefore, be a critical tool for biodiversity conservation. However, it is not clear what impacts of wild ungulates or livestock are tolerable or desirable in forests set aside for conservation or restoration. The primary aim of the proposed systematic review is to clarify how the diversity of plants and invertebrates is affected by manipulation of the grazing/ browsing pressure by livestock or wild ungulates. The ultimate purpose of the review is to investigate whether such manipulation is useful as a means of conserving or restoring biodiversity in forest set-asides.

Methods: The review will examine primary field studies of how fencing or other kinds of manipulation of the grazing/browsing pressure by livestock or wild ungulates affects plants or invertebrates. We will consider studies made in boreal or temperate forests anywhere in the world, incorporating investigations made not only in protected areas but also in stands under commercial management. Non-intervention or alternative levels of grazing pressure will be used as comparators. Relevant outcomes include abundance, diversity and composition of plants and invertebrates, tree regeneration, and performance of focal/target species. Relevant studies will mainly be selected from a recent systematic map of the evidence on biodiversity impacts of active management in forest set-asides. A search update will be made with a subset of the search terms used for the systematic map. Searches for additional literature will be made in bibliographies of existing reviews. Relevant studies will be subject to critical appraisal and categorised as having high, medium or low susceptibility to bias. Studies with high susceptibility to bias will be excluded from the review. Useful outcomes and data on interventions and other potential effect modifiers will be extracted from included articles. A narrative synthesis will describe the quality and findings of all studies in the review. Where studies report similar outcomes, meta-analysis will be performed.
\end{abstract}

Keywords: Biodiversity, Forest conservation, Forest restoration, Forest set-aside, Forest reserve, Herbivory, Livestock, Deer, Elk, Moose, Semi-natural habitat, Silvopastoral system, Wood-pasture

\footnotetext{
*Correspondence: claes.bernes@eviem.se

${ }^{1}$ Mistra Council for Evidence-Based Environmental Management,

Stockholm Environment Institute, Box 24218, 10451 Stockholm, Sweden

Full list of author information is available at the end of the article
} 


\section{Background}

Large herbivorous mammals are a natural part of the fauna of most forest regions of the world, except on remote islands. Their abundance and assemblages have been dramatically affected by humans throughout history, however. Major impacts include use of forests for livestock grazing, deliberate introduction of game species, and strongly increased abundance of wild ungulates due to removal of top predators or supplementary forage in agricultural or production-forest landscapes [1-3]. Of these changes, livestock grazing and 'overabundance' of large wild herbivores have long been perceived as conflicting with the aims of both silviculture and forest conservation [3-6].

On the other hand, the importance of herbivory for the maintenance of habitat values (such as structural and compositional diversity) in forest ecosystems has also been recognised [7-10]. For example, livestock-modified ecosystems can help compensate for the loss of open natural habitats in the profoundly transformed European landscapes $[11,12]$. The re-introduction of plains bison to Banff National Park in Canada was partially motivated by the recognition of their importance in maintaining habitat heterogeneity necessary to conserve biodiversity in the park [13]. Managed grazing has also been considered as a tool for restoration of ecosystems that have become degraded due to a lack of wildfire [14]. While grazing-related conservation values are best known in the case of grasslands, the biodiversity importance of grazed, semi-open forests is also increasingly documented [15]. For example, some authors have proposed that, in longgrazed forests, conservation should maintain 'moderate' grazing levels [16].

Management of grazing/browsing in protected areas can, therefore, be a critical tool for biodiversity conservation. Unfortunately, it is not clear what impacts of wild ungulates or livestock are tolerable or desirable in protected forests, except that they should be aligned with conservation targets [17]. Faced with a wide array of management options-from strict protection to a range of interventions [18]-conservation managers need to understand how biodiversity and other conservation values vary with grazing and browsing pressures.

\section{Identification of review topic}

At the request of Swedish stakeholders, who wished to get an overview of the scientific support for various ways of managing protected forests, we recently published a systematic map focused on the effects of active management on biodiversity in forests set aside for conservation or restoration [19]. A systematic map does not synthesise reported results but rather provides a summary of the evidence base, and it can therefore be seen as a first step towards more complete reviews of selected subtopics. A total of 812 studies describing a variety of interventions in temperate or boreal forests were identified as relevant. The map included studies of interventions that could conceivably be utilised in protected areas, whether the study was conducted in a forest set-aside or in forest under commercial management.

Following completion of the systematic map, the effect of forest grazing/browsing was identified as a candidate topic for full systematic review based on a number of key criteria: the presence of sufficient reliable evidence, the relevance of the topic for stakeholders, the applicability of the topic for the Swedish forests, and the added value of a systematic approach to a topic that has so far received attention only via traditional reviews. The topic was proposed and accepted during a meeting of the authors in April 2015.

The studies in the systematic map that dealt with manipulation of grazing or browsing mainly related to exclusion, enclosure or culling of deer and other wild cervids, but they also included studies on traditional forest grazing by cattle, sheep or other livestock. All these types of intervention are highly relevant to forest conservation, and a review of grazing effects has been explicitly proposed by Swedish stakeholders. Foster et al. [20] provide a quantitative review of impacts of large native herbivores on the species richness and abundance of fauna, and there are several narrative reviews of grazing/browsing effects on various aspects of biodiversity (e.g. [21-25]). However, there is still a need for an explicitly practice-oriented analysis that considers biodiversity targets (e.g., increased tree species diversity, forest structural heterogeneity, abundance of plant or invertebrate species of conservation concern, or reduced abundance of weedy or invasive species) relevant to the active-management context (i.e. manipulation of the grazing pressure in boreal and temperate forest set-asides).

\section{Objectives}

The primary aim of the proposed systematic review is to clarify how the diversity of plants and invertebrates is affected by manipulation of the grazing/browsing pressure by livestock or wild ungulates in temperate and boreal forests. Plants are obviously affected by herbivory, directly or indirectly, and the structural diversity of vegetation is an important aspect of habitat value and thus of conservation value in itself. Invertebrates were included as a highly diverse group that is directly dependent on vegetation structure; further, Foster et al. [20] identified them as being particularly sensitive to herbivory. Both plants and invertebrates also include a number of threatened species. 
The ultimate purpose of the review is to investigate whether manipulation of the grazing/browsing pressure is useful as a means of conserving or restoring biodiversity in forest set-asides. Nonetheless, we will assess manipulation impacts as such on plants and invertebrates, rather than the extent to which these impacts are desirable for conservation. We will also include any relevant studies made in forests under commercial management.

The review will follow the guidelines for systematic reviews in environmental management issued by the collaboration for environmental evidence [26].

\section{Primary question}

What are the impacts of manipulating the pressure of grazing and browsing by livestock or wild ungulates on plants and invertebrates in temperate and boreal forests?

\section{Components of the primary question}

Population: Temperate and boreal forests.

Intervention: Manipulation of the pressure of grazing and browsing by livestock or wild ungulates.

Comparator: Non-intervention or alternative levels of intervention.

Outcomes: $\quad$ Abundance, diversity and composition of plants and/or invertebrates.

Tree regeneration.

Performance (e.g. growth, reproduction) of focal/target species (individual plant or invertebrate species that the intervention was intended to benefit or control).

\section{Methods}

\section{Selection of studies identified in the systematic map}

Most of the evidence on which this systematic review will be based is included in the recently completed systematic map of biodiversity impacts of active management in forest set-asides [19]. Of the 812 studies in the map, 149 reported on how plants or invertebrates were affected by manipulation of grazing or browsing. Nearly all of these dealt with grazing/browsing by wild or domesticated ungulates-the few that focused on other herbivores will be excluded from this review.

The systematic map was based on searches using 13 publication databases, 2 search engines, 24 specialist websites and 10 literature reviews. The majority of searches were performed in May-August 2014. In March 2015, a search update was made using Web of Science and Google Scholar.

\section{Search update}

A comprehensive search for additional potentially relevant studies will be made in the bibliographies of existing reviews of forest grazing/browsing.

Furthermore, in order to identify recently published literature, we will perform an additional search update, using the following subset of search terms applied for the systematic map:

\section{Subject:}

Forest type:

Intervention:

Outcomes: 'intervention' and 'outcomes') will be combined using the Boolean operator 'OR'. The four categories will then be combined using the Boolean operator 'AND'. An asterisk $\left.{ }^{*}\right)$ is a 'wildcard' that represents any group of characters, including no character.

Searches for peer-reviewed or grey literature published in 2014 or later will be made in Web of Science and Google Scholar. In the latter case, the first 200 hits (based on relevance) will be examined for appropriate data. No language or document type restrictions will be applied.

\section{Article screening and study inclusion criteria}

Articles will be evaluated for inclusion at three successive levels. First, they will be assessed by title. Next, each article found to be potentially relevant on the basis of title will be judged for inclusion on the basis of abstract. Finally, each article found to be potentially relevant on the basis of abstract will be judged for inclusion based on the full text. At all stages of this screening process, the reviewer will tend towards inclusion in cases of uncertainty. Articles identified by one reviewer as potentially useful based on full text will be assessed by a second reviewer; and care will be taken to ensure reviewers do not assess studies authored by themselves. Final decisions on whether to include doubtful cases will be taken by the review team as a whole. 
A list of studies rejected on the basis of full-text assessment will be provided in an appendix together with the reasons for exclusion.

In order to be included, each study must pass each of the following criteria (a subset of those used for the systematic map):

- Relevant subjects Forests in the boreal or temperate vegetation zones.

Any habitat with a tree layer is regarded as forest, which means that studies of e.g. wooded meadows and urban woodlands may be included.

As an approximation of the boreal and temperate vegetation zones we will use the cold Köppen-Geiger climate zones (the $\mathrm{D}$ zones) and some of the temperate ones (Cfb, $\mathrm{Cfc}$ and $\mathrm{Csb}$ ), as defined by Peel et al. [27]. The other temperate Köppen-Geiger climate zones are often referred to as subtropical and are therefore considered to fall outside the scope of this review.

Nevertheless, forest stands dominated by ponderosa pine (Pinus ponderosa) will be considered as relevant even if located outside the climate zones mentioned above. These forests constitute a well-studied North American habitat type that shares several characteristics with the pine forests in boreal and temperate regions.

- Relevant types of intervention Manipulation of ungulate grazing/browsing pressure, e.g. by fencing or by introduction or culling of ungulates.

Studies of areas where the grazing/browsing pressure varies for reasons other than direct manipulation (e.g. because of natural differences in accessibility or food availability) will not be included.

- Relevant type of comparator Non-intervention or alternative levels of intervention.

Both temporal and spatial comparisons of how grazing/browsing manipulations affect biodiversity are considered to be relevant. This means that we will include both before/after (BA) studies, i.e. comparisons of the same site prior to and following an intervention, and control/impact (CI) studies, i.e. comparisons of treated and untreated sites (or sites that had been subject to different kinds of treatment). Studies combining these types of comparison, i.e. those with a before/after/control/impact (BACI) design, will also be included.

- Relevant types of outcome Abundance, diversity or composition of plants and/or invertebrates (including exotic species); tree regeneration (seedlings and saplings); performance (e.g. growth, reproduction) of focal/target species (individual plant or invertebrate species that the intervention was intended to benefit or control).

- Relevant type of study Primary field studies.

Based on this criterion, we will exclude e.g. simulation studies, review papers and policy discussions.

- Language Full text written in English, French, German, Danish, Norwegian, Swedish, Finnish, Estonian or Russian.

\section{Study quality assessment}

Studies that have passed the relevance criteria described above will be subject to critical appraisal. Based on assessments of their validity, they will be categorised as having high, medium or low susceptibility to bias.

Studies will be excluded from the review due to high susceptibility to bias (low quality) if any of the following factors apply:

- No true replication.

- Methodological description insufficient.

- Intervention and comparator sites not well-matched.

- Severely confounding factors present.

- Outcomes difficult to interpret.

- Intervention data difficult to interpret.

Confounding factors may include interventions performed in addition to manipulation of the grazing pressure. Historically, wood-pastures were often used for multiple purposes that combined grazing with, for example, mowing, acorn collecting, litter raking and field crop cultivation [15]. However, present-day reserve management typically requires separate consideration of each intervention; therefore, studies of such combined activities (even if historically relevant) will be excluded due to severely confounding factors unless the main effect of grazing can be distinguished.

Studies that are not excluded due to low quality will be considered to have medium susceptibility to bias (medium quality) if any of the following factors apply:

- Location of study plots potentially biased.

- BA study (not CI or BACI).

- No data on variance or sample sizes.

- No quantitative data on grazing/browsing pressure.

If none of the above factors apply, the study will be considered to have low susceptibility to bias (high quality).

Detailed reasoning concerning critical appraisal will be recorded in a transparent manner. In general, the quality 
of a study will be assessed by one reviewer, but again, care will be taken to ensure reviewers do not assess studies authored by themselves. The final ruling on doubtful cases will be made by the review team as a whole. Full justification for our decisions on susceptibility to bias will be provided for each study in an appendix to the final report. The significance of quality issues and other limitations of the available data will be considered in our discussion of review findings. In particular, we will discuss how our results may have been affected by the exclusion of studies that are relevant in principle but have high susceptibility to bias, e.g. due to confounding factors or lack of replication.

\section{Data extraction strategy}

Outcome means, measures of variation and uncertainty (standard deviation, standard error, confidence intervals) and sample sizes will be extracted from tables and graphs, using image analysis software when necessary. The selection of outcomes to be extracted from an article will be made by one reviewer. A second reviewer will reassess this selection and perform the actual data extraction, and the records will be double-checked by a third reviewer. Data on interventions and other potential effect modifiers will also be extracted from the included articles.

It may in some cases be useful to ask authors of relevant articles to supply data in digital format. This will primarily be done where useful data have been published in graphs from which they are difficult to extract accurately enough, or when it is known or assumed that considerable amounts of relevant but unpublished data may be available in addition to the published results. If raw data are provided, summary statistics will be calculated by us. Extracted data records will be made available as an additional file.

\section{Potential effect modifiers and reasons for heterogeneity}

To the extent that data are available, the following potential effect modifiers will be considered and recorded for all studies included in this review:

- Geographical coordinates.

- Altitude.

- Climate (and climate change).

- Mean age of forest stand.

- Dominant tree species.

- Forest density (e.g. basal area or overstorey canopy cover).

- Primary target vegetation for grazers/browsers.

- Type of intervention (exclosures, enclosures, culling etc.)

- Duration and seasonality of intervention.

- Size of exclosures/enclosures and/or study sites.

- Size of sampling plots.
- Ungulate grazer/browser species subject to manipulation.

- Grazing/browsing pressure (e.g. no. of animals per $\mathrm{km}^{2}$ ).

- Other interventions at study sites (harvesting, thinning, understorey removal, mowing, burning etc.)

- Landscape aspects (such as degree of isolation).

- History of land use, grazing and protection.

\section{Data synthesis and presentation}

A narrative synthesis of data from all studies included in the review will describe the quality of the results along with the study findings. Tables will be produced to summarise these results. Where studies report similar outcomes, meta-analysis will be performed. In these cases effect sizes will be standardised and weighted appropriately. Judging from the availability of data, meta-analyses are likely to focus on the species richness, abundance and performance of tree seedlings, saplings and field-layer vegetation, and on the species richness and abundance of shrubs and major groups of invertebrates.

Where meta-analysis is possible, it will take the form of random-effects models. Meta-regressions or subgroup analyses of categories of studies will also be performed where sufficient studies report common sources of heterogeneity. Special efforts will be made to analyse how effects depend on the density of grazing/browsing ungulates. Publication-bias and sensitivity analysis will be carried out where possible. Overall management effects will be presented visually in plots of mean effect sizes and variance.

\section{Abbreviations}

BA: before/after; Cl: control/impact; BACl: before/after/control/impact.

\section{Authors' contributions}

The manuscript was drafted by $C B, A L$ and EM. All authors read and approved the final manuscript.

\section{Author details \\ ${ }^{1}$ Mistra Council for Evidence-Based Environmental Management, Stockholm Environment Institute, Box 24218, 10451 Stockholm, Sweden. ${ }^{2}$ Depart- ment of Natural Sciences, Mid Sweden University, 85170 Sundsvall, Sweden. ${ }^{3}$ Metsähallitus Parks \& Wildlife Finland, c/o UEF, P.O. Box 111, 80101 Joensuu, Finland. ${ }^{4}$ School of Forest Sciences, University of Eastern Finland, P.O. Box 111, 80101 Joensuu, Finland. ${ }^{5}$ Institute of Ecology and Earth Sciences, Tartu University, Vanemuise 46, 51014 Tartu, Estonia. ${ }^{6}$ Department of Renewable Resources, University of Alberta, 751 General Services Building, Edmonton AB T6G 2H1, Canada. ${ }^{7}$ Department of Conservation and Research, Bavarian Forest National Park, Freyunger Str. 2, 94481 Grafenau, Germany.}

\section{Acknowledgements}

The authors wish to thank Per Linder, Johan Nitare, Babs Stuiver, Andrew Pullin and three anonymous referees for valuable comments on the manuscript.

\section{Competing interests}

The authors declare that they have no competing interests. 


\section{Funding}

The review will be financed by the Mistra Council for Evidence-Based Environmental Management (EviEM)

Received: 20 April 2016 Accepted: 12 July 2016

Published online: 22 August 2016

\section{References}

1. Putman R. Grazing in temperate ecosystems: Large herbivores and their effects on the ecology of the new forest. London: Croom Helm/Chapman \& Hall; 1986.

2. Coomes D, Allen R, Forsyth D, Lee W. Factors preventing the recovery of New Zealand forests following control of invasive deer. Conserv Biol. 2003;17:450-9.

3. Côté S, Rooney T, Tremblay J, Dussault C, Waller D. Ecological impacts of deer overabundance. Annu Rev Ecol Evol Syst. 2004;35:113-47.

4. Fleischner T. Ecological costs of livestock grazing in western North America. Conserv Biol. 1994;8:629-44.

5. Putman RJ, Moore NP. Impact of deer in lowland Britain on agriculture, forestry and conservation habitats. Mammal Review. 1998;28(4):141-63.

6. Graham RT, Jain TB, Kingery JL. Ameliorating conflicts among deer, elk, cattle and/or other ungulates and other forest uses: A synthesis. Forestry. 2010;83(3):245-55.

7. Genries A, Morin X, Chauchard S, Carcaillet $C$. The function of surface fires in the dynamics and structure of a formerly grazed old subalpine forest. J Ecol. 2009;97(4):728-41.

8. Oldén A, Raatikainen K, Tervonen K, Halme P. Grazing and soil pH are biodiversity drivers of vascular plants and bryophytes in boreal woodpastures. Agric Ecosyst Environ. 2016;222:171-84.

9. Osem Y, Fogel T, Moshe Y, Brant S. Managing cattle grazing and overstorey cover for the conversion of pine monocultues into mixed Mediterranean woodlands. Appl Veg Sci. 2015;18:261-71.

10. Royo AA, Collins R, Adams MB, Kirschbaum C, Carson WP. Pervasive interactions between ungulate browsers and disturbance regimes promote temperate forest herbaceous diversity. Ecology. 2010;91(1):93-105.

11. Riguero-Rodríguez A, McAdam J, Mosquera-Losada M. Agroforestry in Europe: current status and future prospects. Berlin: Springer; 2009.

12. Wallis De Vries M, Bakker J, Van Wieren S. Grazing and conservation management. Dordrecht: Kluwer Academic Publishers; 1998.
13. Canada P. Plains bison re-introduction. 2015. http://www.pc.gc.ca/eng/ pn-np/ab/banff/plan/gestion-management/bison.aspx. Accessed Feb 25, 2016.

14. Harrington J, Kathol E. Responses of shrub midstory and herbaceous layers to managed grazing and fire in a North American savanna (oak woodland) and prairie landscape. Restor Ecol. 2009;17:234-44.

15. Bergmeier E, Petermann J, Schröder E. Geobotanical survey of woodpasture habitats in Europe: diversity, threats and conservation. Biodivers Conserv. 2010;19(11):2995-3014.

16. Mitchell F, Kirby K. The impact of large herbivores on the conservation of semi-natural woods in the British uplands. Forestry. 1990;63:333-53.

17. Reimoser F, Armstrong H, Suchant R. Measuring forest damage of ungulates: what should be considered. For Ecol Manage. 1999;120:47-58.

18. Götmark F. Habitat management alternatives for conservation forests in the temperate zone: review, synthesis, and implications. For Ecol Manage. 2013:306:292-307.

19. Bernes C, Jonsson B, Junninen K, Lõhmus A, Macdonald E, Müller J, et al. What is the impact of active management on biodiversity in forests set aside for conservation or restoration? A systematic map. Environ Evid. 2015;4:25.

20. Foster C, Barton P, Lindenmayer D. Effects of large native herbivores on other animals. J Appl Ecol. 2014;51:929-38.

21. Gill R, Beardall V. The impact of deer on woodlands: the effects of browsing and seed dispersal on vegetation structure and composition. Forestry. 2001;74:209-18.

22. Kirby KJ. The impact of deer on the ground flora of British broadleaved woodland. Forestry. 2001;74(3):219-29.

23. Rooney T, Waller D. Direct and indirect effects of white-tailed deer in forest ecosystems. For Ecol Manage. 2003;181:165-76.

24. Russell F, Zippin D, Fowler N. Effects of white-tailed deer (Odocoileus virginianus) on plants, plant populations and communities: a review. Am Midl Nat. 2001;146:1-26.

25. Suominen O, Danell K. Effects of large herbivores on other fauna. In: Danell K, Bergström R, Duncan P, Pastor J, editors. Large herbivore ecology, ecosystem dynamics and conservation. Cambridge: Cambridge University Press; 2006. p. 383-412.

26. CEE. Guidelines for systematic reviews in environmental management. Version 4.2. Bangor2013.

27. Peel MC, Finlayson BL, McMahon TA. Updated world map of the KöppenGeiger climate classification. Hydrol Earth Syst Sci. 2007;11:1633-44.

\section{Submit your next manuscript to BioMed Central and we will help you at every step:}

- We accept pre-submission inquiries

- Our selector tool helps you to find the most relevant journal

- We provide round the clock customer support

- Convenient online submission

- Thorough peer review

- Inclusion in PubMed and all major indexing services

- Maximum visibility for your research

Submit your manuscript at www.biomedcentral.com/submit
BioMed Central 